\title{
Violencia escolar School violence
}

\author{
Úrsula Zurita Rivera \\ FLACSO-México \\ uzurita@flacso.edu.mx
}

Recibido / received: 11/02/2018

Aceptado / accepted: 10/03/2018

DOI: https://doi.org/10.20318/eunomia.2018.4171

\section{Resumen}

En las sociedades contemporáneas se observa una multiplicidad de acciones emprendidas en numerosos ámbitos y sectores, bajo la responsabilidad de un amplio abanico de actores de diferente escala, con el fin de prevenir, atender y eliminar la violencia escolar. Dados los graves efectos que este problema provoca no solo entre los individuos e instituciones directamente involucradas, sino también en el fortalecimiento democrático y el ejercicio pleno de los derechos humanos, la prevención de la violencia en las escuelas es una tarea apremiante. Frente al despliegue masivo de estrategias de intervención, a los abundantes estudios realizados en distintos campos disciplinares y a su incorporación en diversas agendas de políticas públicas, es escaso el análisis de las implicaciones que esas estrategias tienen en los principios de autoridad, legalidad y legitimidad en las escuelas. Emprender esta tarea es fundamental, ya que buena parte de las rutas trazadas en el presente para afrontar la violencia escolar -como la creación y aplicación de leyes específicas, el rediseño de acuerdos nacionales, lineamientos para el sistema educativo, manuales para la convivencia hasta los reglamentos escolares, entre otros instrumentos- han afectado dichos principios desconociendo los resultados y efectos que tienen en la conformación, reproducción e, incluso, perpetuación de la violencia escolar.

\section{Palabras clave}

Violencia escolar, autoridad, legalidad, legitimidad, reglamentos escolares, fragmentación de la autoridad.

\begin{abstract}
In contemporary societies, a multiplicity of actions has been taken in different areas and sectors under the responsibility of a wide range of actors on different scales, in order to prevent, attend and eliminate school violence. Since the serious effect of this problem, not only among the individuals and institutions directly involved, but also for the strengthening of democracy and the full exercise of human rights, combating violence in schools is an urgent task. Despite of the massive deployment of intervention strategies, the numerous studies carried out in different academic fields and their inclusion on policy agenda, the implications that these strategies have on the principles of authority, legality and legitimacy in schools has barely been analyzed. This task is essential, given that many of the paths outlined currently to address school violence such as the creation and application of specific laws, the redesign of national agreements, the guidelines for the education system, the manuals for school coexistence, among other instruments- have affected these principles and have ignored the results and implications that they have in the conformation, reproduction and even perpetuation of this problem.
\end{abstract}


Keywords
School violence, authority, legality, legitimacy, school rules, fragmentation of authority.

SUMARIO. 1. Introducción. 2. La violencia escolar respecto a los principios de autoridad, legalidad y legitimidad. 3. Reflexiones finales.

\section{Introducción}

Desde fines del siglo $X X$ se ha advertido el despliegue de políticas, programas, proyectos e incluso legislaciones en diversas regiones y continentes del mundo que han volcado al sector educativo en la prevención y eliminación de la violencia escolar, con especial interés en los niveles que abarcan desde la infancia a la adolescencia. El conocimiento acumulado acerca de la violencia escolar, así como el impacto y resultados de las múltiples estrategias de intervención implementadas por parte de un abanico amplio de actores, merece ser sometido a una indagación sistemática y periódica sobre las consecuencias inesperadas $y$, muchas veces, no deseadas que se desprenden de esas iniciativas (Zurita, 2013 y 2016). Esta tarea es fundamental cuando se piensa que el origen y perduración de la violencia escolar están vinculados con las maneras en que se han construido y consolidado los principios de autoridad, legalidad y legitimidad en las instituciones educativas, y las formas en que dichas estrategias los interpelan y trastocan. Se sabe que, en general, las numerosas estrategias de intervención han corrido a paralelas a profundos cambios en los que las escuelas y los sistemas educativos no han estado al margen, y cuyo sustento proviene de diferentes discursos y proyectos políticos.

Para los propósitos de este documento, conviene resaltar aquellos que han estado comprometidos con los procesos de transformación democrática, descentralización, pluralización y gobernanza desplegados en los países occidentales, sobre todo en las décadas de los años ochenta y noventa del siglo pasado (Aguilar, 2010; Selee, 2011). Es ahí donde se sitúan los numerosos esfuerzos desplegados para incorporar al currículo oficial, a los planes y programas de estudio y a la vida escolar diaria, los programas para la educación ciudadana, la cultura de la legalidad, la formación cívica y ética, la educación en y para los derechos humanos, entre otros (EURYDICE, 2005 y 2012). Paradójicamente, desde el polo opuesto se han impulsado políticas y programas que, tratando de responder al agudo incremento del crimen organizado, el narcotráfico, la delincuencia común y la inseguridad experimentados en varias regiones del mundo -como América Latina y Norteamérica, han formulado y puesto en marcha conservadoras y autoritarias políticas de seguridad nacional (OEA, 2008). De éstas, se han desprendido iniciativas específicas, como los programas Tolerancia Cero o Mano Dura, dirigidos a las escuelas para prevenir y eliminar la violencia suscitada en su interior y en su entorno inmediato, acrecentada por la inseguridad, consumo y venta de drogas, pandillerismo y el uso ilegal de armas, entre otros problemas. Además de esas explicaciones se observan otras de carácter cultural, donde el incremento de la violencia escolar es adjudicado al cambio de valores y comportamientos en las sociedades, a las transformaciones experimentados por las familias y, sin duda, al despegue del uso de las tecnologías de la información y comunicación en todos los ámbitos cotidianos de la vida individual y social. Entre ambas visiones antagónicas, hay un punto en común que merece ser puesto de relieve, y es que la escuela es concebida como una institución que debe responder a las exigencias que las sociedades le imponen, cualesquiera que éstas sean (Zurita, 2013). 
Por ahora, es necesario reflexionar sobre cómo se han trastocado los principios de autoridad, legitimidad y legalidad, a propósito de las acciones desplegadas en contra de la violencia, con el objetivo de examinar algunos de sus efectos en la consecución de los objetivos planteados para impulsar y fortalecer la convivencia escolar libre de violencia. Al hacerlo, se identificarán varios rasgos importantes que distinguen la proliferación reciente de las diversas expresiones de violencia en los centros educativos. Entre estos, cabe destacar la multiplicación de actores externos a las escuelas y a los sistemas educativos que, al gozar de cierta autoridad y legalidad, aunque con grados disímiles de legitimidad, han formulado e implementado numerosas iniciativas encaminadas a diversos propósitos.

Uno de esos propósitos está vinculado con la creación de una normatividad escolar más efectiva que ponga fin a este problema público. El interés por la normatividad escolar fue cobrando cada vez mayor relevancia, entre otras razones por la publicación de una investigación sobre la violencia escolar en América Latina y el Caribe, promovida por Naciones Unidas, donde se señaló que los reglamentos escolares "no están concebidos como pactos de convivencia" (Eljach, 2011, p. 110). Sin ser una característica exclusiva de nuestra región, Sonia Eljach, autora del estudio, subrayó una realidad ampliamente conocida por la gran mayoría de los centros escolares: los reglamentos suelen ser un listado de prohibiciones y sanciones fundamentalmente para el alumnado, cuya creación, difusión y aplicación está en manos de los supervisores, directores y docentes.

Frente a este panorama, suele pasar desapercibido que se ha configurado un quiebre respecto a las formas en que tradicionalmente se "resolvían" los problemas de violencia escolar. Este fenómeno tiene que ver con la fragmentación e, incluso, pulverización de la autoridad escolar. Un rasgo esencial de toda figura de autoridad es precisamente la capacidad que tiene para tomar decisiones que, además, sean reconocidas y aceptadas por la comunidad respectiva. Este aspecto a primera vista anodino, revela que las instituciones educativas históricamente adoptaron principios relativos a quiénes y cómo se debía decidir, a fin de preservar el orden, el control y la disciplina escolar. Esto es, los reglamentos ayudaban a establecer las condiciones necesarias para la enseñanza y el aprendizaje (Zurita, 2016).

En respuesta a la preocupación por la normatividad escolar destinada a prevenir la violencia en los centros educativos, se ha desplegado en la gran mayoría de los países latinoamericanos una multiplicidad de instrumentos de diferente estatus jurídico, que han sido creados desde fuera y desde adentro de los sistemas educativos, y cuya implementación en el espacio escolar ha sido impulsada por una variedad de actores (Carrasco, et. al., 2012; Litichever, 2012; Magendzo, et. al., 2013; Zurita, 2011). Lo que importa subrayar es que esta tendencia ha fragmentado la autoridad que ostentan todos aquellos individuos e instituciones que están facultadas legalmente para imponer normas y sanciones en los centros educativos. Es decir, en sociedades como las de América Latina y el Caribe, la violencia en las escuelas conduce a la ruptura del principio de "la legitimidad del poder", debido a que la actuación de los directores y docentes que tradicionalmente habían tenido la autoridad y legitimidad para construir las reglas e imponer sanciones en los centros educativos se ve amenazada por la intervención de otros actores externos a las escuelas y a los sistemas educativos, provocando que ese comportamiento no se ajuste a lo establecido por normas (Marcilla, 2014, p. 177). A ello se suma que los contextos latinoamericanos se caracterizan por procesos de transformación democráticos paradójicos, puesto que, por un lado, hay notorios avances en la defensa y promoción de los derechos humanos, y, por otra, persisten agudas y permanentes amenazas por los crecientes grados de violencia social, violencia de género, feminicidios, entre otros. Estos son asuntos que, aunque para podrían 
parecer lejanos a las escuelas y sus comunidades, tienen una notoria presencia en los contenidos de las políticas y programas para la prevención de la violencia en las escuelas de nuestra región.

Así, en el acontecer cotidiano de las escuelas, los directores y docentes están cada vez más confundidos por la multiplicidad de instrumentos que proceden del exterior y al pretender que sean aplicados en la escuela, sienten que su autoridad, legitimidad y legalidad están siendo cuestionados por actores externos que, en su opinión, desconocen la naturaleza, dinámicas y dispositivos que determinan la emergencia de la violencia en los centros educativos. Además de la confusión entre los integrantes de las comunidades escolares respecto a la legalidad y legitimidad de las autoridades que les imponen decisiones, se ha documentado la construcción de un doble discurso que, de nueva cuenta, le resta sentido a los proyectos que buscan disminuir la violencia mediante iniciativas acordes con los principios democráticos y los derechos humanos. Para desarrollar este planteamiento, en las siguientes páginas se examinan algunos resultados e implicaciones que las iniciativas para prevenir y eliminar la violencia escolar han provocado en los principios de autoridad, legalidad y legitimidad en los centros escolares.

2. La violencia escolar respecto a los principios de autoridad, legalidad y legitimidad La creación de la normatividad escolar, cuyos procesos formales e informales determinan quiénes, cómo, cuándo, por qué y para qué la hacen, está estrechamente vinculada con varios planteamientos clásicos de las ciencias sociales y las humanidades. Por ahora, conviene centrarse en la perspectiva que provee el Derecho sobre los principios de autoridad, legalidad y legitimidad, que todo conjunto de normas expresa independientemente del estatus que dicho ordenamiento posea. En términos concretos, estos principios ayudan a responder cuestiones fundamentales en las sociedades. Por ejemplo, el principio de legalidad conduce a preguntarse cómo es la actuación de la autoridad que construye esas normas respecto a otras figuras de autoridad, así como frente a la comunidad donde esas normas se aplicarán; y, en segundo lugar, indica cómo es respecto al conjunto de normas pre-existentes, las cuales establecen límites de diferente tipo para su comportamiento en relación con la construcción de normas. El principio de legalidad permite responder qué tanto se respetan dichas normas y qué tanto son un referente para la actuación de los sujetos involucrados (Laveaga, 1999).

Ahora bien, por investigaciones empíricas realizadas en México y por la revisión documental de casos latinoamericanos (Zurita, 2016, 2013, 2011), es factible observar con gran nitidez que la autoridad escolar está disputada por actores externos al sistemas educativo -como los Congresos, las Comisiones de Derechos Humanos, la Suprema Corte de Justicia, las Procuradurías Generales de Justicia, las Secretarías de Salud-, quienes en la última década han incrementado notoriamente su intervención en la prevención, atención, eliminación e, incluso, sanción de la violencia escolar a partir de las iniciativas de gran escala (leyes específicas, acuerdos nacionales, protocolos, tesis de la Suprema Corte de Justicia), que se van decantando hasta llegar a replantear el sentido, contenido y significado de acciones en la escala micro, materializadas en instrumentos que regulan la convivencia cotidiana (reglamentos escolares). Por ejemplo, en el primer caso, la emisión de leyes en materia de seguridad, acoso, violencia y convivencia escolar tiene procesos singulares que, desde su origen, propician la configuración de sentidos e implicaciones diferentes para los principios de legalidad y legitimidad a la hora de abordar este problema público. El mosaico latinoamericano expresa este hecho y, de manera precisa, el caso mexicano lo ilustra cuando se advierte que los resultados que buscan tener las leyes en las materias señaladas arriba no son los deseados. Todo empieza con la forma en que se hacen las leyes y esa manera varía de acuerdo con 
el tipo de Estado que sea. Por ejemplo, Colombia es un Estado soberano, democrático y unitario organizado políticamente en 32 departamentos descentralizados y el Distrito Capital, donde reside el gobierno nacional; mientras que México es una república democrática, representativa y federal, compuesta por 32 entidades federativas. Eso implica que la manera en que se hacen las leyes en ambos países es disímil, pero en todos los casos se registran implicaciones generales y otras particulares, según los contextos educativos y políticos asociados a las figuras de autoridad que, de acuerdo con el poder que legalmente poseen, tienen la capacidad no sólo de hacerlas sino de que sean acatadas por las comunidades respectivas.

A ello se suma que cada país tiene su historia política y, del mismo modo, su sistema educativo también la tiene. En este sentido vale la pena recordar que en los años noventa se impulsaron reformas educativas en buena parte de América Latina que, entre otros propósitos, abogaban por la descentralización educativa y la democratización de los sistemas escolares (López, 2007). Cada país latinoamericano vivió estos procesos dentro de otros más generales orientados a la transformación democrática. En este sentido, en México, a pesar de que formalmente ha sido una república federal desde épocas remotas, las entidades no practicaban tales facultades en el ámbito educativo hasta la reforma educativa lanzada en 1992 con el Acuerdo Nacional para la Modernización de la Educación Básica, cuando fueron creados los sistemas educativos estatales. Esto condujo a que las autoridades de las entidades tuviesen la atribución de tomar decisiones y emprender acciones en materia educativa, con la excepción de asuntos salariales del magisterio, el presupuesto educativo y manejo de gran parte de los recursos y, por último, del contenido de los planes y programas educativos.

Por otra parte, una de las expresiones más relevantes del creciente interés público por la violencia escolar en México es, precisamente, la existencia de alrededor de una treintena de leyes sobre seguridad, acoso, violencia y convivencia escolar, aprobadas en casi todas las entidades del país en el lapso de una década, aproximadamente. Este es un hecho sin precedentes en la historia del sistema educativo mexicano, no sólo por las leyes en sí mismas, por los tópicos a los cuales se dirigen, por el número de leyes aprobadas o por las formas en que se hicieron, sino también por los procesos relativos a su instrumentación y aplicación, que han tratado de impulsarse en los sistemas educativos y las escuelas. Aunque se han configurado experiencias singulares de cada entidad, lo cierto es que hasta ahora los resultados han sido escasos en todas ellas (Zurita, 2016, 2013). La complejidad y especificidad requiere un análisis detallado, pues son diversos factores, y no solo educativos ni escolares, los que han determinado estas consecuencias.

Pero, si la atención se concentra en la creación masiva de dichas leyes, podría pensarse que es un indicio de la sensibilidad de los legisladores, que actuaron de forma expedita ante un conjunto de complejos y graves fenómenos en nuestra sociedad (Cárdenas y Cárdenas, 2010). Sin embargo, esta reacción inusual no puede entenderse si se ignora que está situada, a su vez, dentro de los cambios registrados en la manera de hacer las leyes en México durante los últimos veinte años (Béjar, 2012). De acuerdo con Luisa Béjar, esta transformación se debe fundamentalmente a dos transiciones registradas en la reciente historia política nacional concernientes, por un lado, al paso de un arreglo autoritario a uno democrático; y, por otro, de un régimen centralizado a otro descentralizado. En el primer caso, una de sus implicaciones más relevantes ha sido el incremento de agentes que pueden incidir en el debate y aprobación en los Congresos. En el segundo, se ha vuelto más difícil el proceso legislativo y más inciertos sus resultados. De este modo, la época donde el presidente decidía de forma total o parcialmente unilateral lo más importante, mientras que los Congresos solo obedecían, parece haber concluido. Los actores 
subnacionales se han multiplicado y tienen mayor capacidad y poder para incidir en los procesos legislativos.

Ese contexto pudo haber incidido de forma importante en la promulgación de la treintena de leyes específicas en casi todas las entidades de México. Sin embargo, a diferencia de otros países latinoamericanos, en México no se ha logrado el consenso necesario en el Congreso de la Unión para contar con una ley federal, a pesar de las iniciativas que han sido presentadas. No obstante, ello no ha sido obstáculo para que otras agencias gubernamentales y organismos públicos aprueben instrumentos para prevenir y eliminar la violencia escolar, como fue el caso de la Suprema Corte y las Comisiones de Derechos Humanos, por ejemplo. Ahora bien, en otros países latinoamericanos también han sido creados instrumentos de diferente estatus jurídico de carácter nacional, federal y/o subnacional para prevenir, atender y eliminar la violencia escolar. Entre éstos se encuentran Argentina (Ley 26.892 para la Promoción de la convivencia y el abordaje de la conflictividad en las instituciones educativas), Bolivia (Ley 548), Chile (Ley sobre violencia escolar y Política Nacional de Convivencia Escolar 2015/2018), Colombia (Ley 1620 Por la cual se crea el Sistema Nacional de Convivencia Escolar y Formación para el Ejercicio de los Derechos Humanos, la Educación para la Sexualidad y la Prevención y Mitigación de la Violencia Escolar) y Costa Rica (Ley $N^{\circ} 7476$, Ley contra Hostigamiento o Acoso Sexual en el Empleo y la Docencia). Estas acciones, emprendidas por los Congresos, por varias agencias gubernamentales y por los ministerios de educación, paulatinamente han sido incorporadas a los sistemas educativos, transformando los sentidos, alcances y significados de la normatividad materializada en los reglamentos escolares. Por ahora, en Argentina, Chile y México han sido documentadas estas experiencias. Si bien la naturaleza de estos procesos varía de país a país, la percepción predominante entre los integrantes de las comunidades escolares es que "los cambios son impulsados desde fuera y desde arriba de las escuelas"; lo cual, inevitablemente, genera un cuestionamiento a la legalidad y legitimidad de aquellos actores que tomaron esas decisiones.

Los análisis publicados en estos temas indican que, lejos de promover la conformación de espacios seguros y de ambientes propicios para la enseñanza y el aprendizaje, dichos instrumentos jurídicos tienen poca o débil consistencia con los principios del derecho a la educación y el régimen internacional de derechos humanos (Henry, 2009; Carrasco, et. al., 2012; Litichever, 2012; Magendzo, et. al., 2013; Ochoa y Díez-Martínez, 2013; Landeros y Chávez, 2015; Zurita, 2016, 2013). La consecuencia de esto es, por ejemplo, la inexistente o limitada participación de la comunidad escolar en su construcción, especialmente de los principales involucrados -los estudiantes-; el predominio de un acentuado enfoque punitivo; la incorporación excesiva e ilimitada de sanciones; el poco conocimiento y la limitada difusión de las normas entre los diferentes integrantes de las comunidades, y la carencia de los elementos centrales del debido proceso en su aplicación (Landeros y Chávez, 2015; Zurita, 2016). Este panorama se agrava todavía más porque no son pocos los instrumentos jurídicos que desconocen la naturaleza de la violencia en las escuelas y de sus diferentes expresiones, que la hacen un fenómeno incomparable respecto a otros tipos de violencia que suceden en otros terrenos (Gómez y Zurita, 2013; Zurita, 2013). Además, las leyes, los acuerdos nacionales, las tesis emitidas por la Suprema Corte de Justicia en esta materia, los marcos de convivencia escolar y otros instrumentos jurídicos tampoco consideran el importante papel que tiene el diseño institucional y organizacional de las escuelas en aspectos sustanciales concernientes a quiénes y cómo toman las normas destinadas a mantener el control, la disciplina y el orden. Tal es el caso de los lineamientos, que han sido propagados en los últimos años para construir los reglamentos escolares en las escuelas mexicanas de nivel básico (Zurita, 2016). 
Por ello es indispensable saber lo que ocurre en las acciones de escala micro dirigidas hacia las escuelas. Donde, después de un conjunto abigarrado de mediaciones organizacionales e institucionales propias de los sistemas educativos, se busca plasmar aquellos cambios en la vida escolar cotidiana. El gran problema es que esos cambios impulsados por leyes, acuerdos, marcos de convivencia y otros instrumentos conciben a la violencia escolar como un problema social. Al partir desde esta perspectiva, se crean iniciativas que no llegan a la esencia de las instituciones escolares (Dubet, 2004, 2010). Con ello, se ignora el hecho esencial de que, según la investigación educativa, la violencia escolar es una manifestación de otros fenómenos que ocurren a su interior (Zurita, 2016).

Los reglamentos escolares, así como las valoraciones construidas por los diferentes integrantes de las comunidades escolares, poseen una gran relevancia en el acontecer cotidiano de las escuelas. Es decir, además de que dichos reglamentos articulan su funcionamiento y organización e inciden en el logro escolar de los alumnos, también influyen en su desempeño social en tanto miembros de una comunidad respecto a la cual construyen vínculos de pertenencia e identidad (Appleton et al, 2008: 369). Ahora bien, en el marco de la institucionalización y consolidación que experimentó en la última década el área de investigación abocada a la convivencia, disciplina y violencia escolar en Latinoamérica, se aprecia un repunte reciente en trabajos relacionados con el estudio de las normas escolares en general y de los reglamentos escolares en particular (Zurita, 2016). Buena parte de este repunte se explica por la asunción, un tanto extendida, de que la violencia escolar y, especialmente, su reciente y acentuado incremento, obedece a la falta de normas y, sobre todo, de sanciones aplicables en las escuelas. Frente a ese supuesto, las posturas se multiplican, pues para algunos no es la carencia sino la existencia de normas que poco sirven para construir, establecer y aplicar sanciones lo suficientemente severas que, de forma efectiva y eficiente, contribuyan a reducir la violencia y consigan que los padres, madres y/o tutores sean lo suficientemente estrictos para emprender en el hogar acciones disciplinarias acordes con las impuestas en las escuelas. Algunos más consideran que las normas son incongruentes e ilógicas, dado que se orientan hacia cuestiones que actualmente no tienen relación estrecha con las causas de la violencia ni del conflicto, como la apariencia, la vestimenta y la higiene personal, y cuya utilidad existía tiempos atrás cuando respondían a los esfuerzos por imponer cierta homogeneidad y asegurar la disciplina y el control escolar. Otros especialistas, autoridades y representantes de organismos civiles opinan que, para tener mayor legitimidad, las normas tendrían que ser construidas democráticamente entre los distintos integrantes de las comunidades escolares. Como se puede advertir, las visiones sobre las normas y los reglamentos son diversas y en ellas se expresan tanto posturas autoritarias como democráticas, así como otras que combinan factores de ambas.

Mientras tanto, el alumnado es educado con la plena convicción de que las normas que realmente se aplican día a día a través de los reglamentos no tienen nada que ver con normas generales, prospectivas, estables, claras, transparentes e imparciales (Vázquez, 2012). El alumnado sabe por su propia experiencia que los procesos de construcción y los contenidos de los reglamentos son imprecisos, ignorados y restringidos. No ignoran tampoco que su aplicación es discrecional, parcial y privada (Zurita, 2016).

En general, a pesar de los esfuerzos y recomendaciones por construir "verdaderos pactos de convivencia escolar" (Eljach, 2011), continúa prevaleciendo la idea de que los reglamentos son un instrumento crucial e indispensable para la disciplina, el orden y el control escolar. En este contexto, la disciplina ha sido un instrumento estratégico e imprescindible para silenciar voces, implantar la autoridad, 
uniformar a los individuos, aniquilar la diferencia y, desde ahí, garantizar el funcionamiento de una institución autoritaria, vertical y jerárquica donde unos mandan y otros obedecen, donde unos saben y otros aprenden.

No obstante, las numerosas acciones para prevenir y eliminar la violencia en las escuelas cifran sus buenos resultados en varios factores, entre los cuales sobresale el uso efectivo de reglamentos escolares y la aplicación de sanciones a quienes cometan actos indebidos. Pero, conviene subrayar que los reglamentos revelan las concepciones, posturas y valoraciones de una comunidad escolar, frente a las formas en que los principios asociados a la legalidad y legitimidad de aquellos concebidos como las autoridades (directores, docentes o, bien otras figuras externas a las escuelas y a los sistemas educativos) se plasman en decisiones y acciones concretas.

En este marco, la proliferación de programas para la prevención de la violencia escolar acordes con la perspectiva de Tolerancia Cero, caracterizados por los enfoques punitivos, autoritarios y discrecionales, han recibido numerosas descalificaciones. La oleada de críticas negativas se debe a que estas iniciativas no ayudan a prevenir y atender problemas específicos, sino que amenazan la garantía y ejercicio pleno del derecho a la educación. Las medidas disciplinarias que proponen, como las suspensiones y expulsiones, propician la disminución de tiempo destinado a la instrucción, el incremento de las dificultades académicas, la desvinculación/desafiliación escolar y el aumento del riesgo al rezago y abandono escolar.

Aunado a ello, se tiene ampliamente documentado el componente de discriminación, racismo y exclusión en la aplicación de dichas medidas, que suelen ser aplicadas en mayor medida y severidad a ciertos grupos poblacionales como los que tienen alguna discapacidad, los que pertenecen a un estatus socioeconómico bajo, los indígenas, los afrodescendientes, los que tienen un bajo rendimiento y aprovechamiento, los migrantes, entre otros. En contraste, los programas que han tenido resultados más satisfactorios para revertir los procesos institucionales de las escuelas que perpetúan diversas expresiones de violencia escolar, han optado por crear reglamentos y normas basadas en principios de justicia restaurativa. Este tipo de justicia le da mayor relevancia a la noción de involucramiento social y menos a la de control social orientado a la construcción, mantenimiento y reformulación de las relaciones entre los individuos que forman parte de un contexto social determinado (Zurita, 2016).

La cuestión es que en la gran mayoría de las estrategias lanzadas para prevenir la violencia en las escuelas se termina apelando a los principios de legalidad y legitimidad que tienen las autoridades escolares y educativas para decidir la creación, difusión y aplicación de las normas y los reglamentos escolares. Con todo, a pesar de los esfuerzos recientes, como algunas reformas educativas que impulsan la democratización de los sistemas educativos y la autonomía de la gestión escolar, la participación de los múltiples integrantes de comunidades escolares continúa estando al margen de la toma de decisión de asuntos prioritarios, como la creación, uso y aplicación de los reglamentos escolares.

En resumen, lo que se observa es que la autoridad de las escuelas, que tradicionalmente encarnaban los directores y docentes, está disputada por otras figuras externas que, desde numerosos polos, les encomiendan a las escuelas diversos propósitos, no siempre congruentes entre sí, ni tampoco con los objetivos depositados en las instituciones educativas. Así, se les exige a las escuelas que sean más eficientes y eficaces en la reducción de la violencia escolar cuando, 
paradójicamente, se les han reducido las responsabilidades y atribuciones para lograrlo.

\section{Reflexiones finales}

En este documento se expusieron varias implicaciones que las acciones para combatir la violencia han provocado en los principios de autoridad, legalidad y legitimidad en los centros escolares. Este hecho inusitado requiere ser estudiado detalladamente debido a que, como bien lo han documentado desde tiempos remotos, dichos principios han estructurado no solo el funcionamiento cotidiano, sino la propia esencia de la escuela como una institución propia de la modernidad.

Con todo, los reglamentos escolares son construcciones histórica y culturalmente situadas. Su análisis implica considerar la activación de estos procesos en los ámbitos en los cuales se inscribe su creación, difusión, uso y aplicación. Estos procesos solían estar limitados al espacio escolar y al sistema educativo. Sin embargo, a pesar de que esa realidad cada vez se disipa más, los integrantes de las comunidades escolares tienen un papel importante en esas acciones, aunque su intervención no siempre sea legítima, legal ni abiertamente reconocida, pues persisten limitadas oportunidades y experiencias de participación escolar en asuntos relevantes. (Zurita, 2016).

La disputa por la autoridad, la legalidad y la legitimidad en las escuelas se está configurando en los diferentes niveles institucionales y organizacionales de los sistemas educativos y también en áreas gubernamentales de otros sectores, órdenes y poderes, espacios civiles e internacionales, como lo muestran los países latinoamericanos que han compartido esta experiencia. A nivel macro y desde una visión optimista, se ha gestado una democratización, descentralización y pluralización en el espacio de las políticas educativas; lo cual se vive de distinto modo según el régimen político, el tipo de estado, el grado de democratización y descentralización y la consolidación de la ciudadanía, entre otros factores (Selee, 2011). Esto se podría acercar a un esquema de gobernanza, que implicaría que las metas, al ser establecidas colectivamente, sean establecidas por múltiples actores, que participarían en la elaboración y puesta en práctica de las políticas públicas dirigidas a problemas contemporáneos -como la violencia escolar-, que por su complejidad requieren ser atendidos mediante acciones integrales llevadas a cabo con la coresponsabilidad de actores situados en diferentes niveles y espacios (Aguilar, 2010). El lado negativo de este escenario es que se ha fragmentado la autoridad y las decisiones tomadas son numerosas, diferentes y hasta opuestas, no solo en lo concerniente a lo que se debe hacer para prevenir la violencia sino, incluso, a qué se entiende por violencia escolar, cómo debe ser sancionada y quién lo debe hacer. Desde este acercamiento, la autoridad, legalidad y legitimidad que se les reconocía a las escuelas y a los sistemas educativos para atender este problema, está siendo disputada en el presente por parte de actores que históricamente no solían involucrarse en esos asuntos. Con ello, se han creado y profundizado vacíos y contradicciones respecto a qué le corresponde hacer a las escuelas y a sus autoridades para prevenir, atender y eliminar la violencia escolar. Al no ser tomada en cuenta esta implicación no deseada, buena parte de las iniciativas encaminadas a la consecución de esos propósitos, están enfrentando desde su comienzo, más riesgos de fracasar. 
Bibliografía

AGUILAR, L. F. (2010), Gobernanza: El nuevo proceso de gobernar, Fundación Friederich Naumann para la Libertad, México.

APPLETON, J., J., CHRISTENSON, S. L. y FURLONG, M. J. (2008), "Student engagement with school: Critical conceptual and methodological issues of the construct", Psychology in the Schools, Vol. 45 (5), pp. 369-386.

BÉJAR, L. (2012), “¿Quién legisla en México? Descentralización y poder legislativo en México", Revista Mexicana de Sociología, 74, núm. 4, octubre-diciembre, pp. 619-647.

CÁRDENAS, S. y CÁRDENAS, M. (2010), La participación del poder legislativo en la definición de la política educativa en México., CIDE, Colecc. Documentos de Trabajo 253, México

CARRASCO, C., LÓPEZ, V. y ESTAY, C. (2012), "Análisis crítico de la Ley de Violencia Escolar en Chile", Psicoperspectivas, Individuo y Sociedad, vol. 11, núm. 2, pp. $31-55$.

DUBET, F. (2004), "Conflictos de normas y ocaso de la institución", Estudios sociológicos, vol. XXII, núm. 64, enero-abril, pp. 3-24.

DUBET, F. (2010), "Crisis de la transmisión y declive de la institución", Política y Sociedad, vol. 47, núm. 2, pp. 15-25.

ELJACH, S. (2011), Violencia escolar en América Latina y el Caribe: superficie y fondo, PLAN INTERNACIONAL Y UNICEF, Panamá.

GÓMEZ, A. y ZURITA, U. (2013), "El estudio de la violencia escolar, orígenes y tendencias". En: FURLAN, A. y SPITZER, T. C. (Coord. Gral.), Convivencia, disciplina y violencia en las escuelas 2002 - 2011. México, COMIE - ANUIES, pp. 183-222.

HENRY, S. (2009), "School violence beyond Columbine. A complex problem in need of an interdisciplinary analysis", American Behavioral Scientis, Vol. 52, num. 9, pp. 1246-1265.

LANDEROS, L. y CHÁVEZ, C. (2015), Convivencia y disciplina en la escuela. Análisis de reglamentos escolares de México, INEE, México.

LAVEAGA, G. (1999), Cultura de la legalidad, Serie Estudios Jurídicos número 8, Instituto de Investigaciones Jurídicas (UNAM), México.

LITICHEVER, L. (2012), "¿Qué se regula hoy en las escuelas? Una mirada sobre las prescripciones de los reglamentos de convivencia", Revista Iberoamericana de Educación, ONU OEI, núm. 59, disponible en la siguiente dirección electrónica: http://rieoei.org/historico/deloslectores/4839Litichever.pdf (fecha de consulta: 15 de febrero de 2018).

LÓPEZ, N. (2007), Las nuevas leyes de educación América Latina. Una lectura a la luz del panorama social y educativo de la región, Instituto Internacional de Planeamiento de la Educación IIPE-UNESCO, Campaña Latinoamericana por el Derecho a la Educación, Buenos Aires.

MAGENDZO, A., TOLEDO, M. y GARCÍA, V. (2013), "Descripción y análisis de la Ley sobre violencia escolar (20.536): dos paradigmas antagónicos", Estudios Pedagógicos, Vol. 39, núm. 1, pp. 377 - 391.

MARCILLA, G. (2014), "Imperio de la ley", Eunomía. Revista en Cultura de la Legalidad, núm. 5, Septiembre-Febrero, pp. 177-185.

OCHOA, A. y DIEZ-MARTíNEZ, E. (2013), "El reglamento escolar como eje de análisis de la convivencia en la escuela", Ensaio: aval. pol. públ. Educ., v. 21, n. 81, out./dez. pp. 667-684.

ORGANIZACIÓN DE LOS ESTADOS AMERICANOS (OEA) (2008), La Seguridad Pública en las Américas: retos y oportunidades. Washington D. C.: OEA documentos oficiales.

RED EUROPEA DE INFORMACIÓN EN EDUCACIÓN (EURYDICE) (2012), La educación para la ciudadanía en Europa. España: Eurydice. 
RED EUROPEA DE INFORMACIÓN EN EDUCACIÓN (EURYDICE) (2005), La educación para la ciudadanía en el contexto escolar europeo, España: Eurydice.

SELEE, A. (2011), Decentralization, Democratization, and Informal Power in Mexico, Pennsylvania State University Press, Pennsylvania.

VAZQUEZ, R. (2012), "Legalidad y laicidad", Eunomía. Revista en Cultura de la Legalidad, $\mathrm{n}^{\circ} 3$, septiembre 2012-febrero 2013, pp. 141-149.

ZURITA, U. (2011), "Las escuelas mexicanas y la legislación sobre convivencia, seguridad y violencia", Revista Educación y Territorio, vol. 1, número 2, pp. 1936.

ZURITA, U. (2013), "Políticas, programas, legislación, proyectos y acciones gubernamentales y no gubernamentales". En: FURLAN, A. y SPITZER, T. C. (Coord. Gral.), Convivencia, disciplina y violencia en las escuelas 2002 - 2011. México, COMIE - ANUIES, pp. 457-520.

ZURITA, U. (2016), No gritar, no correr, no empujar. Las miradas, voces y acciones de los estudiantes y los docentes del DF respecto a las normas, UNAM, México. 